\title{
Author Correction: Identification of multiple risk loci and regulatory mechanisms influencing susceptibility to multiple myeloma
}

\author{
Molly Went et al.
}

Correction to: Nature Communications; https://doi.org/10.1038/s41467-018-04989-w, published online 13 September 2018

The original version of this Article contained an error in the spelling of a member of the PRACTICAL Consortium, Manuela GagoDominguez, which was incorrectly given as Manuela Gago Dominguez. This has now been corrected in both the PDF and HTML versions of the Article. Furthermore, in the original HTML version of this Article, the order of authors within the author list was incorrect. The PRACTICAL consortium was incorrectly listed after Richard S. Houlston and should have been listed after Nora Pashayan. This error has been corrected in the HTML version of the Article; the PDF version was correct at the time of publication.

Published online: 10 January 2019

Molly Went ${ }^{1}$, Amit Sud (1) ${ }^{1}$, Asta Försti ${ }^{2,3}$, Britt-Marie Halvarsson ${ }^{4}$, Niels Weinhold ${ }^{5,6}$, Scott Kimber ${ }^{7}$, Mark van Duin ${ }^{8}$, Gudmar Thorleifsson', Amy Holroyd', David C. Johnson (1) ${ }^{7}, \mathrm{Ni} \mathrm{Li}^{1}$, Giulia Orlando?, Philip J. Law (1) ${ }^{1}$, Mina Ali ${ }^{4}$, Bowang Chen², Jonathan S. Mitchell', Daniel F. Gudbjartsson (10,10, Rowan Kuiper ${ }^{8}$, Owen W. Stephens ${ }^{5}$, Uta Bertsch ${ }^{2,11}$, Peter Broderick (10) ${ }^{1}$, Chiara Campo ${ }^{2}$, Obul R Bandapalli (10 ${ }^{2}$, Hermann Einsele ${ }^{12}$, Walter A. Gregory ${ }^{13}$, Urban Gullberg ${ }^{4}$, Jens Hillengass ${ }^{6}$, Per Hoffmann ${ }^{14,15}$, Graham H. Jackson ${ }^{16}$, Karl-Heinz Jöckel ${ }^{17}$, Ellinor Johnsson ${ }^{4}$, Sigurður Y. Kristinsson ${ }^{18}$, Ulf-Henrik Mellqvist ${ }^{19}$, Hareth Nahi ${ }^{20}$, Douglas Easton (10) 21,22 , Paul Pharoah (1) ${ }^{21,22}$, Alison Dunning ${ }^{21}$, Julian Peto ${ }^{23}$, Federico Canzian (10 ${ }^{24}$, Anthony Swerdlow ${ }^{1,25}$, Rosalind A. Eeles (1) ${ }^{1,26}$, Zsofia Kote-Jarai , Kenneth Muir (1) ${ }^{27,28,}$ Nora Pashayan (10 21,29, The PRACTICAL consortiumJolanta Nickel ${ }^{6}$, Markus M. Nöthen ${ }^{14,30}$, Thorunn Rafnar ${ }^{9}$, Fiona M. Ross ${ }^{31}$, Miguel Inacio da Silva Filho², Hauke Thomsen², Ingemar Turesson ${ }^{32}$, Annette Vangsted ${ }^{33}$, Niels Frost Andersen ${ }^{34}$, Anders Waage ${ }^{35}$, Brian A. Walker ${ }^{5}$, Anna-Karin Wihlborg ${ }^{4}$, Annemiek Broyl ${ }^{8}$, 
Faith E. Davies ${ }^{5}$, Unnur Thorsteinsdottir ${ }^{9,36}$, Christian Langer ${ }^{37}$, Markus Hansson (1) ${ }^{4,32}$, Hartmut Goldschmidt ${ }^{6,11}$, Martin Kaiser ${ }^{7}$, Pieter Sonneveld ${ }^{8}$, Kari Stefansson ${ }^{9}$, Gareth J. Morgan ${ }^{5}$, Kari Hemminki ${ }^{2,3}$, Björn Nilsson ${ }^{4,38}$ \& Richard S. Houlston (10 ${ }^{1,7}$

${ }^{1}$ Division of Genetics and Epidemiology, The Institute of Cancer Research, London SW7 3RP, UK. ² German Cancer Research Center, 69120 Heidelberg, Germany. ${ }^{3}$ Center for Primary Health Care Research, Lund University, SE-205 02 Malmo, Sweden. ${ }^{4}$ Hematology and Transfusion Medicine, Department of Laboratory Medicine, BMC B13, Lund University, SE-221 84 Lund, Sweden. ${ }^{5}$ Myeloma Institute for Research and Therapy, University of Arkansas for Medical Sciences, Little Rock, AR 72205, USA. ${ }^{6}$ Department of Internal Medicine V, University of Heidelberg, 69117 Heidelberg, Germany. ${ }^{7}$ Division of Molecular Pathology, The Institute of Cancer Research, London SW7 3RP, UK. ${ }^{8}$ Department of Hematology, Erasmus MC Cancer Institute, 3075 EA Rotterdam, The Netherlands. ${ }^{9}$ deCODE Genetics, Sturlugata 8, IS-101 Reykjavik, Iceland. ${ }^{10}$ School of Engineering and Natural Sciences, University of Iceland, IS-101 Reykjavik, Iceland. ${ }^{11}$ National Centre of Tumor Diseases, 69120 Heidelberg, Germany. ${ }^{12}$ University Clinic of Würzburg, 97080 Würzburg, Germany. ${ }^{13}$ Clinical Trials Research Unit, University of Leeds, Leeds LS2 9PH, UK. ${ }^{14}$ Institute of Human Genetics, University of Bonn, D-53127 Bonn, Germany. ${ }^{15}$ Division of Medical Genetics, Department of Biomedicine, University of Basel, 4003 Basel, Switzerland. ${ }^{16}$ Royal Victoria Infirmary, Newcastle upon Tyne NE1 4LP, UK. ${ }^{17}$ Institute for Medical Informatics, Biometry and Epidemiology, University Hospital Essen, University of Duisburg-Essen, Essen D-45147, Germany. ${ }^{18}$ Department of Hematology, Landspitali, National University Hospital of Iceland, IS-101 Reykjavik, Iceland. ${ }^{19}$ Section of Hematology, Sahlgrenska University Hospital, Gothenburg 41345 , Sweden. ${ }^{20}$ Center for Hematology and Regenerative Medicine, SE-171 77 Stockholm, Sweden. ${ }^{21}$ Centre for Cancer Genetic Epidemiology, Department of Oncology, University of Cambridge, Cambridge CB1 8RN, UK. ${ }^{22}$ Centre for Cancer Genetic Epidemiology, Department of Public Health and Primary Care, University of Cambridge, Cambridge CB1 8RN, UK. ${ }^{23}$ Department of Non-Communicable Disease Epidemiology, London School of Hygiene and Tropical Medicine, London WC1E 7HT, UK. ${ }^{24}$ Genomic Epidemiology Group, German Cancer Research Center (DKFZ), Heidelberg 69120, Germany. ${ }^{25}$ Division of Breast Cancer Research, The Institute of Cancer Research, London SW7 3RP, UK. ${ }^{26}$ Royal Marsden NHS Foundation Trust, Fulham Road, London SW3 6JJ, UK. ${ }^{27}$ Institute of Population Health, University of Manchester, Manchester M13 9PL, UK. ${ }^{28}$ Warwick Medical School, University of Warwick, Coventry CV4 7AL, UK. ${ }^{29}$ Department of Applied Health Research, University College London, London WC1E 7HB, UK. ${ }^{30}$ Department of Genomics, Life \& Brain Center, University of Bonn, D-53127 Bonn, Germany. ${ }^{31}$ Wessex Regional Genetics Laboratory, University of Southampton, Salisbury SP2 8BJ, UK. ${ }^{32}$ Hematology Clinic, Skåne University Hospital, SE-221 85 Lund, Sweden.

${ }^{33}$ Department of Haematology, University Hospital of Copenhagen at Rigshospitalet, Blegdamsvej 9, DK-2100 Copenhagen, Denmark.

${ }^{34}$ Department of Haematology, Aarhus University Hospital, Tage-Hansens Gade 2, DK-8000 Aarhus C, Denmark. ${ }^{35}$ Department of Cancer Research and Molecular Medicine, Norwegian University of Science and Technology, Box 8905N-7491 Trondheim, Norway. ${ }^{36}$ Faculty of Medicine, University of Iceland, IS-101 Reykjavik, Iceland. ${ }^{37}$ Department of Internal Medicine III, University of Ulm, D-89081 Ulm, Germany. ${ }^{38}$ Broad Institute, 7 Cambridge Center, Cambridge, MA 02142, USA. These authors contributed equally: Molly Went, Amit Sud, Asta Försti, Britt- Marie Halvarsson, Niels Weinhold. These authors jointly supervised the work: Hartmut Goldschmidt, Kari Stefansson, Gareth J. Morgan, Björn Nilsson, Kari Hemminki, Richard S. Houlston. Deceased: Brian E. Henderson. The original article can be found online at https://doi.org/10.1038/s41467-018-04989-w.

\section{The PRACTICAL consortium}

Brian E. Henderson ${ }^{39}$, Christopher A. Haiman ${ }^{39}$, Sara Benlloch ${ }^{1}$, Fredrick R. Schumacher ${ }^{40,41}$, Ali Amin Al Olama ${ }^{22,42}$, Sonja I. Berndt ${ }^{43}$, David V. Conti ${ }^{39}$, Fredrik Wiklund ${ }^{44}$, Stephen Chanock ${ }^{43}$, Victoria L. Stevens ${ }^{45}$, Catherine M. Tangen ${ }^{45}$, Jyotsna Batra ${ }^{46,47}$, Judith Clements ${ }^{46,47}$, Henrik Gronberg ${ }^{44}$, Johanna Schleutker ${ }^{48,49,50}$, Demetrius Albanes ${ }^{43}$, Stephanie Weinstein ${ }^{43}$, Alicja Wolk ${ }^{51}$, Catharine West ${ }^{52}$, Lorelei Mucci ${ }^{53}$, Géraldine Cancel-Tassin ${ }^{54,55}$, Stella Koutros ${ }^{43}$, Karina Dalsgaard Sorensen ${ }^{56,57}$, Eli Marie Grindedal ${ }^{58}$, David E. Neal ${ }^{59,60}$, Freddie C. Hamdy ${ }^{61,62}$, Jenny L. Donovan ${ }^{63}$, Ruth C. Travis ${ }^{64}$, Robert J. Hamilton ${ }^{65}$, Sue Ann Ingles', Barry Rosenstein ${ }^{66,67}$, Yong-Jie Lu ${ }^{68}$, Graham G. Giles ${ }^{69,70}$, Adam S. Kibel ${ }^{71}$, Ana Vega ${ }^{72}$, Manolis Kogevinas ${ }^{73,74,75,76}$, Kathryn L. Penney ${ }^{77}$, Jong Y. Park ${ }^{78}$, Janet L. Stanford 79,80 , Cezary Cybulski ${ }^{81}$, Børge G. Nordestgaard ${ }^{82,83}$, Hermann Brenner ${ }^{84,85,86}$, Christiane Maier $^{87}$, Jeri Kim ${ }^{88}$, Esther M. John ${ }^{89,90}$, Manuel R. Teixeira ${ }^{91,92}$, Susan L. Neuhausen ${ }^{93}$, Kim De Ruyck ${ }^{94}$, Azad Razack ${ }^{95}$, Lisa F. Newcomb ${ }^{79,96}$, Davor Lessel ${ }^{97}$, Radka Kaneva ${ }^{98}$, Nawaid Usmani ${ }^{99,100}$, Frank Claessens ${ }^{101}$, Paul A. Townsend ${ }^{102}$, Manuela Gago-Dominguez ${ }^{103,104}$, Monique J. Roobol ${ }^{105}$, Florence Menegaux ${ }^{106}$, Kay-Tee Khaw ${ }^{107}$, Lisa Cannon-Albright ${ }^{108,109}$, Hardev Pandha ${ }^{110}$ \& Stephen N. Thibodeau ${ }^{110}$

\footnotetext{
${ }^{39}$ Department of Preventive Medicine, Keck School of Medicine, University of Southern California/Norris Comprehensive Cancer Center, Los Angeles 90033 CA, USA. ${ }^{40}$ Department of Epidemiology and Biostatistics, Case Western Reserve University, Cleveland 44106 OH, USA. ${ }^{41}$ Seidman Cancer Center, University Hospitals, Cleveland 44106 OH, USA. ${ }^{42}$ Department of Clinical Neurosciences, University of Cambridge, Cambridge CB2 1TN, UK. ${ }^{43}$ Division of Cancer Epidemiology and Genetics, National Cancer Institute, NIH, Bethesda 20892 MD, USA.

${ }^{44}$ Department of Medical Epidemiology and Biostatistics, Karolinska Institute, Stockholm SE-177 77, Sweden. ${ }^{45}$ Epidemiology Research Program, American Cancer Society, 250 Williams Street, Atlanta 30303 GA, USA. ${ }^{46}$ Australian Prostate Cancer Research Centre-QId, Institute of Health and Biomedical Innovation and School of Biomedical Science, Queensland University of Technology, Brisbane 4059 QLD, Australia. ${ }^{47}$ Translational Research Institute, Brisbane 4102 QLD, Australia. ${ }^{48}$ Department of Medical Biochemistry and Genetics, Institute of Biomedicine, University of
} 
Turku, Turku Fl-20520, Finland. ${ }^{49}$ Tyks Microbiology and Genetics, Department of Medical Genetics, Turku University Hospital, Turku Fl-20520, Finland. ${ }^{50}$ BioMediTech, University of Tampere, Tampere 33100, Finland. ${ }^{51}$ Division of Nutritional Epidemiology, Institute of Environmental Medicine, Karolinska Institutet, Solna SE-171 77, Sweden. ${ }^{52}$ Institute of Cancer Sciences, University of Manchester, Manchester Academic Health Science Centre, Radiotherapy Related Research, The Christie Hospital NHS Foundation Trust, Manchester M13 9NT, UK. ${ }^{53}$ Department of Epidemiology, Harvard School of Public Health, Boston 02115 MA, USA. ${ }^{54}$ CeRePP, Pitie-Salpetriere Hospital, Paris 75013, France. ${ }^{55}$ UPMC Univ Paris 06, GRC No. 5 ONCOTYPE-URO, CeRePP, Tenon Hospital, Paris 75020, France. ${ }^{56}$ Department of Molecular Medicine, Aarhus University Hospital, Aarhus 8000, Denmark. ${ }^{57}$ Department of Clinical Medicine, Aarhus University, Aarhus 8000, Denmark. ${ }^{58}$ Department of Medical Genetics, Oslo University Hospital, Oslo N-0424, Norway. ${ }^{59}$ University of Cambridge, Department of Oncology, Addenbrooke's Hospital, Cambridge CB2 OQQ, UK. ${ }^{60}$ Cancer Research UK Cambridge Research Institute, Ka Shing Centre, Cambridge CB2 ORE, UK. ${ }^{61}$ Nuffield Department of Surgical Sciences, University of Oxford, Oxford OX3 9DU, UK. ${ }^{62}$ Faculty of Medical Science, University of Oxford, John Radcliffe Hospital, Oxford OX3 9DU, UK. ${ }^{63}$ School of Social and Community Medicine, University of Bristol, Bristol BS8 2PS, UK. ${ }^{64}$ Cancer Epidemiology, Nuffield Department of Population Health, University of Oxford, Oxford OX3 7LF, UK. ${ }^{65}$ Department of Surgical Oncology, Princess Margaret Cancer Centre, Toronto M5G 2M9, Canada. ${ }^{66}$ Department of Radiation Oncology, Icahn School of Medicine at Mount Sinai, New York 10029 NY, USA. ${ }^{67}$ Department of Genetics and Genomic Sciences, Icahn School of Medicine at Mount Sinai, New York, NY 10029, USA. ${ }^{68}$ Centre for Molecular Oncology, Barts Cancer Institute, Queen Mary University of London, John Vane Science Centre, London EC1M 6BQ, UK. ${ }^{69}$ Cancer Epidemiology \& Intelligence Division, The Cancer Council Victoria, Melbourne 3004 VIC, Australia. ${ }^{70}$ Centre for Epidemiology and Biostatistics, Melbourne School of Population and Global Health, The University of Melbourne, Melbourne 3053 VIC, Australia. ${ }^{71}$ Division of Urologic Surgery, Brigham and Womens Hospital, Boston 02115 MA, USA. ${ }^{72}$ Fundación Pública Galega de Medicina Xenómica-SERGAS, Grupo de Medicina Xenómica, CIBERER, IDIS, Santiago de Compostela 15782, Spain. ${ }^{73}$ Centre for Research in Environmental Epidemiology (CREAL), Barcelona Institute for Global Health (ISGlobal),

Barcelona 60803, Spain. ${ }^{74}$ CIBER Epidemiología y Salud Pública (CIBERESP), Madrid 28029, Spain. ${ }^{75}$ IMIM (Hospital del Mar Research Institute), Barcelona 08003, Spain. ${ }^{76}$ Universitat Pompeu Fabra (UPF), Barcelona 08002, Spain. ${ }^{77}$ Channing Division of Network Medicine, Department of Medicine, Brigham and Women's Hospital/Harvard Medical School, Boston 02115 MA, USA. ${ }^{78}$ Department of Cancer Epidemiology, Moffitt Cancer Center, Tampa 33612 FL, USA. ${ }^{79}$ Division of Public Health Sciences, Fred Hutchinson Cancer Research Center, Seattle 98109 WA, USA. ${ }^{80}$ Department of Epidemiology, School of Public Health, University of Washington, Seattle 98195 WA, USA. ${ }^{81}$ International Hereditary Cancer Center, Department of Genetics and Pathology, Pomeranian Medical University, Szczecin 70-001, Poland. ${ }^{82}$ Faculty of Health and Medical Sciences, University of Copenhagen, Copenhagen 1165, Denmark. ${ }^{83}$ Department of Clinical Biochemistry, Herlev and Gentofte Hospital, Copenhagen University Hospital, Herlev 2900, Denmark. ${ }^{84}$ Division of Clinical Epidemiology and Aging Research, German Cancer Research Center (DKFZ), Heidelberg 69120, Germany. ${ }^{85}$ German Cancer Consortium (DKTK), German Cancer Research Center (DKFZ), Heidelberg 69120, Germany. ${ }^{86}$ Division of Preventive Oncology, German Cancer Research Center (DKFZ) and National Center for Tumor Diseases (NCT), Heidelberg 69120, Germany. ${ }^{87}$ Institute for Human Genetics, University Hospital Ulm, Ulm 89081, Germany. ${ }^{88}$ Department of Genitourinary Medical Oncology, The University of Texas M. D. Anderson Cancer Center, Houston 77030 TX, USA. ${ }^{89}$ Cancer Prevention Institute of California, Fremont 94538 CA, USA. ${ }^{90}$ Department of Health Research \& Policy (Epidemiology) and Stanford Cancer Institute, Stanford University School of Medicine, Stanford 94305 , CA, USA. ${ }^{91}$ Department of Genetics, Portuguese Oncology Institute of Porto, Porto 4200-072, Portugal. ${ }^{92}$ Biomedical Sciences Institute (ICBAS), University of Porto, Porto 4200-072, Portugal. ${ }^{93}$ Department of Population Sciences, Beckman Research Institute of the City of Hope, Duarte 91016 CA, USA. ${ }^{94}$ Ghent University, Faculty of Medicine and Health Sciences, Basic Medical Sciences, Ghent 9000, Belgium. ${ }^{95}$ Department of Surgery, Faculty of Medicine, University of Malaya, Kuala Lumpur 50603, Malaysia. ${ }^{96}$ Department of Urology, University of Washington, Seattle 98105 , WA, USA. ${ }^{97}$ Institute of Human Genetics, University Medical Center Hamburg-Eppendorf, Hamburg 20246, Germany. ${ }^{98}$ Molecular Medicine Center, Department of Medical Chemistry and Biochemistry, Medical University, Sofia 1431, Bulgaria. ${ }^{99}$ Department of Oncology, Cross Cancer Institute, University of Alberta, Edmonton T6G 2R3 Alberta, Canada. ${ }^{100}$ Division of Radiation Oncology, Cross Cancer Institute, Edmonton T6G 1 Z2 $\mathrm{AB}$, Canada. ${ }^{101}$ Molecular Endocrinology Laboratory, Department of Cellular and Molecular Medicine, KU Leuven, Leuven 3000, Belgium.

${ }^{102}$ Institute of Cancer Sciences, Manchester Cancer Research Centre, University of Manchester, Manchester Academic Health Science Centre, St. Mary's Hospital, Manchester M13 9WL, UK. ${ }^{103}$ Genomic Medicine Group, Galician Foundation of Genomic Medicine, Instituto de Investigacion Sanitaria de Santiago de Compostela (IDIS), Complejo Hospitalario Universitario de Santiago, Servicio Galego de Saúde, SERGAS, Santiago De Compostela 15706, Spain. ${ }^{104}$ University of California San Diego, Moores Cancer Center, La Jolla 92093 CA, USA. ${ }^{105}$ Department of Urology, Erasmus University Medical Center, Rotterdam 3015, The Netherlands. ${ }^{106}$ Cancer \& Environment Group, Center for Research in Epidemiology and Population Health (CESP), INSERM, University Paris-Sud, University Paris-Saclay, Villejuif F-94805, France. ${ }^{107}$ Clinical Gerontology Unit, University of Cambridge, Cambridge CB2 2QQ, UK. ${ }^{108}$ Division of Genetic Epidemiology, Department of Medicine, University of Utah School of Medicine, Salt Lake City 84132 UT, USA. ${ }^{109}$ George E. Wahlen Department of Veterans Affairs Medical Center, Salt Lake City 84148 UT, USA. ${ }^{110}$ The University of Surrey, Guildford, Surrey GU2 7XH, UK 